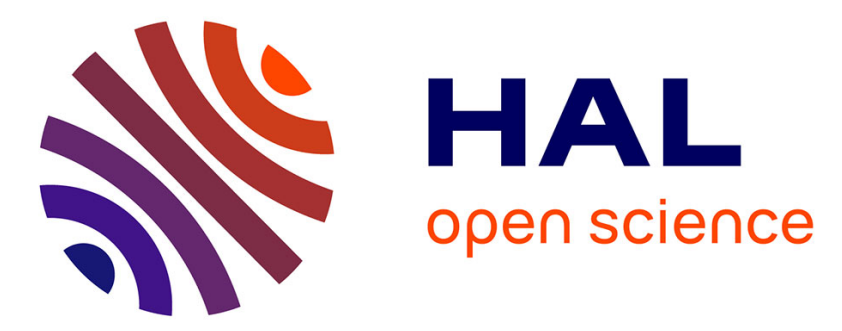

\title{
3D Analytical Model for a Tubular Linear Induction Generator in a Stirling Cogeneration System
}

Pierre Francois, Isabelle Garcia Burel, Hamid Ben Ahmed, Laurent Prevond, Bernard Multon

\section{- To cite this version:}

Pierre Francois, Isabelle Garcia Burel, Hamid Ben Ahmed, Laurent Prevond, Bernard Multon. 3D Analytical Model for a Tubular Linear Induction Generator in a Stirling Cogeneration System. IEEE IEMDC 2007, May 2007, ANTALYA, Turkey. pp.392-397. hal-00676232

\section{HAL Id: hal-00676232 https://hal.science/hal-00676232}

Submitted on 4 Mar 2012

HAL is a multi-disciplinary open access archive for the deposit and dissemination of scientific research documents, whether they are published or not. The documents may come from teaching and research institutions in France or abroad, or from public or private research centers.
L'archive ouverte pluridisciplinaire HAL, est destinée au dépôt et à la diffusion de documents scientifiques de niveau recherche, publiés ou non, émanant des établissements d'enseignement et de recherche français ou étrangers, des laboratoires publics ou privés. 


\title{
3D Analytical Model for a Tubular Linear Induction Generator in a Stirling cogeneration system
}

\author{
P. François, I. Garcia Burrel, H. Ben Ahmed, L. Prévond, B. Multon \\ SPEELabs/SATIE (UMR CNRS 8029), Ecole Normale Supérieure de Cachan \\ pierre.francois@cnam.fr
}

\begin{abstract}
This article sets forth a 3D analytical model of a tubular linear induction generator. In the intended application, the slot and edge effects as well as induced current penetration phenomena within the solid mover cannot be overlooked. Moreover, generator optimization within the present context of cogeneration has necessitated a systemic strategy. Reliance upon an analytical modeling approach that incorporates the array of typically-neglected phenomena has proven essential to offering greater computational and analytical flexibility. This article will describe the electromagnetic model of the generator and draw comparisons with a finite element model, in addition to identifying the elements of equivalent electrical diagram and displaying results from the multi-objective optimization study performed using a genetic algorithm.
\end{abstract}

Index Terms - Linear Induction Generator, edge effects, Stirling engine, multi-objective optimization

\section{INTRODUCTION}

$T_{\mathrm{a}}^{\mathrm{h}}$ he production of electrical energy must be integrated into an optimal strategy with respect to sustainable development goals. As such, it would appear that a sizable and growing share of all electricity consumption stems, to an increasing extent, from a decentralized cogeneration-based (electricity and heat) or trigeneration-based (electricity, heat and air conditioning) production system [1].

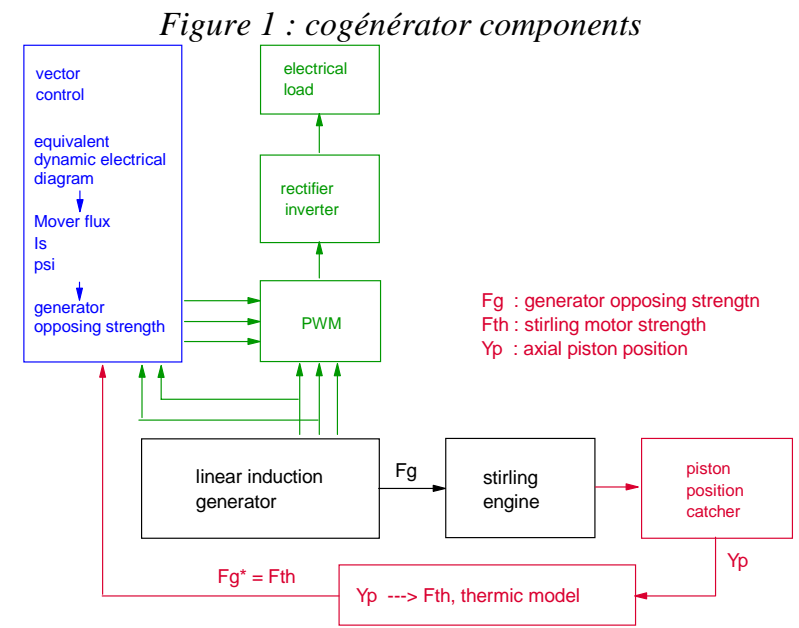

The purpose of the present study lies within this specific scope and proposes exploring new solutions for the autonomous production of electricity in cogeneration mode. While lowpower cogenerators do exist, their flexibility in making thermal and electric power adjustments is inadequate and their life cycle generally proves too short with respect to the type of applications involved.

The cogenerator studied herein, composed of a Stirling engine coupled directly to a linear generator, is intended to be economical, quiet, 'clean', compact and robust. The associated electrical system for performing the electromechanical conversion of energy has been designed as a tubular induction generator with a solid mover. The global optimization of this engine-generator-electronics-load-control cogeneration system requires, for each subsystem, a set of flexible models adapted to a systemic approach. Against this backdrop, an analytical modeling strategy thus seems best suited for our generator. However, given the highly-dynamic operating mode and maximum allowable dimensions, the model must be built to incorporate skin effects along with edge effects.

\section{DESCRIPTION OF THE COGENERATOR}

As shown in Figure 2, the drive assembly is composed of two Stirling engines working in opposition [2].

The thermal and electric parts are thus very tightly integrated, which serves to constitute a compact electric generating set. The Stirling external-combustion engine is particularly welladapted to the cogeneration mode, as its pumping chamber may be assimilated with a boiler [3]. Besides this aptitude for cogeneration operations, such a configuration also proves beneficial through: simplifying the kinematics by introducing linear motion; taking advantage of external combustion in the steady state; and facilitating the guidance of mobile parts thanks to the induction system. Such characteristics would suggest much longer life cycles with much less maintenance.

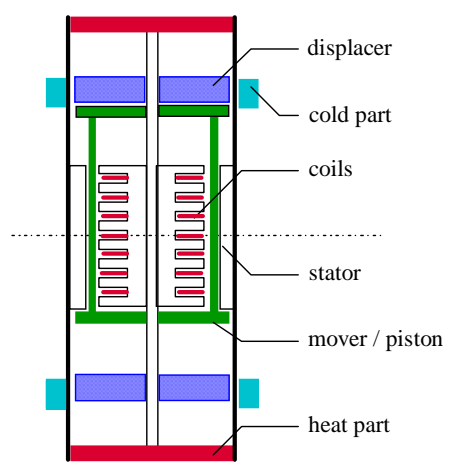

Figure 2: Layout of the cogenerator

The generator studied is of the tubular linear induction type (see Fig. 3). The mover comprises the drive engine pistons and is made of a solid, conductive non-magnetic material like aluminum.

The operating mode (very fast alternating motions), along with the unique structure and its dimensions, make it necessary to develop specific models that incorporate the edge effect as well as dependence of the generator's 
electromagnetic parameters on frequency variations (i.e. a dynamic model).

To proceed with this approach, the following hypotheses have been adopted:

- All materials used are isotropic;

- Magnetic saturation never occurs;

- The spatial distribution of primary windings (stator) is presumed to be symmetrical three-phase.

Moreover, the frequency-based approach defined herein has been based on a power supply composed of three-phase sinusoidal currents balanced at a frequency of $\omega_{s}$. This step will subsequently lead to easily identifying components of the equivalent electrical diagram and thereby extend the design to other types of non-sinusoidal power supplies.

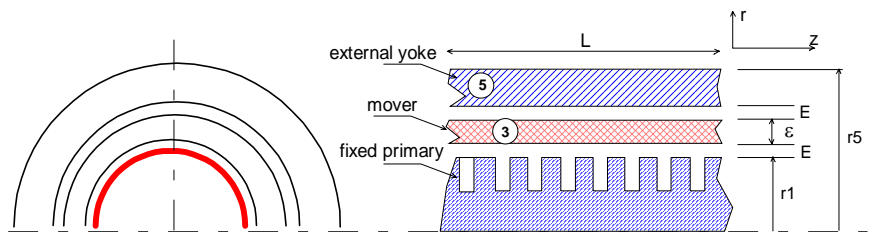

Figure 3: Generator design geometry

The search for generator performance (in terms of axial force, electromagnetic power, losses, etc.) necessitates solving Maxwell's equations, i.e.:

$\overrightarrow{\operatorname{rot}(\overrightarrow{\mathrm{H}})}=\overrightarrow{\mathrm{J}}$

$\overrightarrow{\mathrm{E}}=-\frac{\partial \overrightarrow{\mathrm{B}}}{\partial \mathrm{t}}$

(2), with:

$\overrightarrow{\mathrm{B}}=\mu \cdot \overrightarrow{\mathrm{H}}$ (permeability $\mu$ is assumed to be constant)

$\vec{J}=\sigma(\vec{E}+v \wedge \vec{B})$

$\overrightarrow{\mathrm{B}}=\overrightarrow{\operatorname{rot}(\overrightarrow{\mathrm{A}})}$

In order to describe the analytical modeling steps carried out more simply, we adopted a gradual approach in which both the edge and slot effects are first neglected $[4,5,6]$ and then get taken into account via a sequence of appropriate modulation functions.

\section{GENERATOR ALYTICAL MODEL}

Let's begin by considering an infinite-length geometry along the $z$-axis.

\section{A. Infinite length analytical model}

\section{1) Slotless stator}

The primary current is first modeled by means of a surface current sheet with a perfectly-sinusoidal distribution given by the following expression (see Fig. 4):

$$
\lambda_{s}(z, t)=\hat{\lambda}_{s} \cdot e^{j\left(k z-\omega_{s} t\right)}
$$

with: $j^{2}=-1, \omega_{\mathrm{s}}$ is the primary (stator) current angular frequency and $k$ a propagation coefficient: $k=\pi / \tau$.

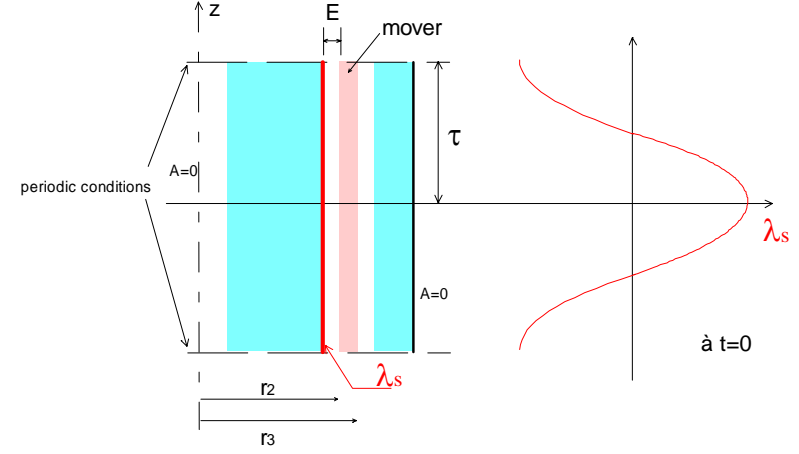

Figure 4: Slotless, infinite-length model

According to our initial hypotheses, the vector potential can be written as:

$\overrightarrow{\mathrm{A}}(\mathrm{z}, \mathrm{r}, \mathrm{t})=\varphi(\mathrm{r}) \mathrm{e}^{\mathrm{j}\left(\mathrm{kz}-\omega_{\mathrm{s}} \mathrm{t}\right)} \overrightarrow{\mathrm{u}}_{\theta}$

A comprehensive understanding of the vector potential at any point within the computation field thus requires determining its radial distribution $\varphi(r)$.

Based on Maxwell's equations and the relation in (5) above, we can derive a quite remarkable differential equation whose general solution consists of a sum of modified Bessel functions of both the $1^{\text {st }}$ and $2^{\text {nd }}$ kind. The vector potential distribution function in each region (index $n$ ) of the computation space can then be expressed in the following general form, equation (8) :

$\mathrm{A}^{[\mathrm{n}]}(\mathrm{z}, \mathrm{r}, \mathrm{t})=\left[\mathrm{X}^{[\mathrm{n}]} \cdot \mathrm{I}_{1}\left(\gamma^{[\mathrm{n}]} \cdot \mathrm{r}\right)+\mathrm{Y}^{[\mathrm{n}]} \cdot \mathrm{K}_{1}\left(\gamma^{[\mathrm{n}]} \cdot \mathrm{r}\right)\right] \cdot \mathrm{e}^{\mathrm{j}\left(\mathrm{kz}-\omega_{\mathrm{s}} \mathrm{t}\right)}$

where for each region [n], we have defined a coefficient $\gamma^{[n]}=\sqrt{k^{2}+j \omega_{s} g{ }^{[n]} \mu^{[n]} \sigma^{[n]}}$.

The coefficients $\mathrm{X}^{[\mathrm{n}]}$ and $\mathrm{Y}^{[\mathrm{n}]}$ are constants determined from the region boundary conditions. $g^{[n]}, \mu^{[n]}$ and $\sigma^{[n]}$ are the slip, magnetic permeability and electrical conductivity of region $[\mathrm{n}]$, respectively.

Based on this solution, it becomes straightforward to deduce, for each region [n], the expressions for both the axial component of the magnetic field and the azimuthal component of the electric field, equations (9a) et (9b) :

$$
\begin{aligned}
& \mathrm{H}^{[\mathrm{nn}]}(\mathrm{z}, \mathrm{r}, \mathrm{t})=\frac{1}{\mu^{[n]}}\left(\frac{\mathrm{A}^{[\mathrm{n}]}}{\mathrm{r}}+\frac{\partial \mathrm{A}^{[\mathrm{n}]}}{\partial \mathrm{r}}\right)=\frac{\gamma^{[\mathrm{n}]}}{\mu^{[n]}}\left[\mathrm{X}^{[\mathrm{nn}]} \cdot \mathrm{I}_{0}\left(\gamma^{[\mathrm{nn}]} \cdot \mathrm{r}\right)-\mathrm{Y}^{[\mathrm{n}]} \cdot \mathrm{K}_{0}\left(\gamma^{[\mathrm{n}]} \cdot r\right)\right] \cdot \mathrm{e}^{\mathrm{j}\left(\mathrm{k}, \mathrm{z}-\mathrm{\omega}_{\mathrm{s}} \mathrm{t}\right)} \\
& \mathrm{E}_{\theta}^{[\mathrm{n}]}(\mathrm{z}, \mathrm{r}, \mathrm{t})=\mathrm{j} \omega_{\mathrm{s}} \mathrm{A}=\mathrm{j} \omega_{\mathrm{s}}\left[\mathrm{X}^{[\mathrm{n}]} \cdot \mathrm{I}_{1}\left(\gamma^{[\mathrm{n}]} \cdot r\right)+\mathrm{Y}^{[\mathrm{n}]} \cdot \mathrm{K}_{1}\left(\gamma^{[\mathrm{n}]} \cdot r\right)\right] \cdot \mathrm{e}^{\mathrm{j}\left(\mathrm{k} \cdot \mathrm{z}-\omega_{\mathrm{s}} \mathrm{t}\right)}
\end{aligned}
$$

The power exchanged between the mover and the fixed parts (expressed in complex values) corresponds to the difference in input and output power through the mover's exterior and interior surfaces $S^{[n]}$, $\mathrm{P}_{\text {tr }}=\mathrm{P}\left(\mathrm{r}_{3}\right)-\mathrm{P}\left(\mathrm{r}_{2}\right)$

These power values correspond to the flux of the Poynting vector through surface $S^{[n]}$ :

$P\left(r^{[n]}\right)=\frac{1}{2} E_{\theta}\left(\gamma[n] \cdot r^{[n]}\right) \cdot H_{z}^{*}\left(\gamma^{[n]} \cdot r^{[n]}\right) \cdot S^{[n]}$ 
Inserting Equations (9a) and (9b) into (10) yields a power function independent of the time of value:

$\mathrm{P}\left(\mathrm{r}^{[\mathrm{n}]}\right)=-\frac{1}{2} \mathrm{j} \omega_{\mathrm{s}} \frac{\gamma^{[\mathrm{n}]}}{\mu^{[\mathrm{n}]}} \cdot\left[\mathrm{X}^{[\mathrm{n}]}\left(\mathrm{I}_{0}+\mathrm{K}_{0} *\right)-\mathrm{Y}^{[\mathrm{n}]}\left(\mathrm{I}_{1}-\mathrm{K}_{1} *\right)\right] \cdot S^{[\mathrm{n}]}$

The real part of $\mathrm{P}_{\mathrm{tr}}$ corresponds to the transmitted electromagnetic power. The axial electromagnetic force $F_{z}$ therefore simply equals:

$$
\mathrm{F}_{\mathrm{z}}=\frac{\mathfrak{R}\left[\overline{\mathrm{P}}\left(\mathrm{r}_{3}\right)-\overline{\mathrm{P}}\left(\mathrm{r}_{2}\right)\right]}{\mathrm{V}_{\mathrm{S}}}
$$

Figure 5 presents, for a given generator geometry, the evolution in axial force vs. rotor current frequency output by the analytical model and the finite element model. These results reveal the very strong correlation between these two models in the case where both edge and slot effects have been neglected
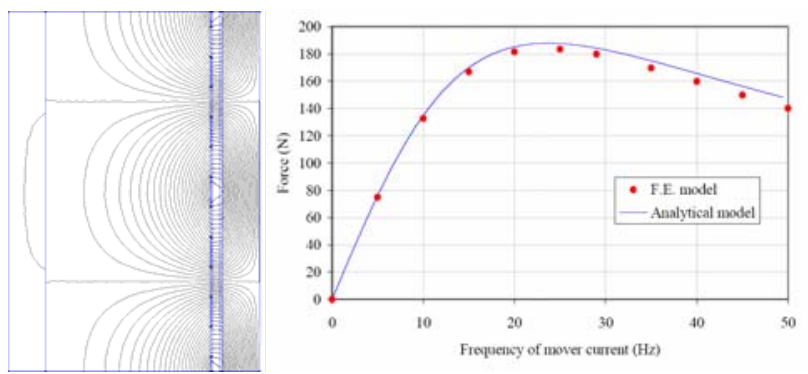

Figure 5: Flux density calculated using finite elements for $v=v_{s}(a)$, and the force developed for a slotless,

infinite-length machine vs. the rotor current frequency (b)

\section{2) Slotted stator}

Slot effects will now be taken into account by focusing on:

1- increase in the actual airgap by means of the Carter coefficient $\mathrm{K}_{\mathrm{c}}$ (see Fig. 6) [7,8];

2- modulation in the current density due to discretization of the primary winding:

$$
\lambda_{s}(z, t)=\hat{\lambda}_{s} \sum_{m=1,5, . .}^{m=\infty} \frac{6}{m \pi} \cdot \sin \left(\frac{m \pi}{12}\right) \cdot e^{j\left(m k z+\chi \omega_{s} t\right)}
$$

where: $\quad \chi=\left\{\begin{array}{l}-1 \text { for } m=1,7, \ldots \\ +1 \text { for } m=5,11, \ldots\end{array}\right.$

The solution, for each harmonic $m$, of Maxwell's equations now enables writing, equation (13):

$A^{[n]}(z, r, t)=\sum_{m=1,5, . .}^{\infty}\left[X_{m}{ }^{[n]} \cdot I_{1}\left(\gamma_{m}{ }^{[n]} \cdot r\right)+Y_{m}{ }^{[n]} \cdot K_{1}\left(\gamma_{m}{ }^{[n]} \cdot r\right)\right] \cdot e^{j\left(k_{m} z+\chi \omega_{s} t\right)}$

with: $\quad \gamma_{\mathrm{m}}^{[\mathrm{n}]}=\sqrt{\mathrm{k}_{\mathrm{m}}{ }^{2}+\mathrm{j} \chi \omega_{\mathrm{s}} \mathrm{g}_{\mathrm{m}}{ }^{[\mathrm{n}]} \mu^{[\mathrm{n}]} \sigma^{[\mathrm{n}]}}, \quad k_{m}=m \pi / \tau$,

$g_{m}=1+\chi m(g-1)$

The synchronous speed for each harmonic of space $m$ equals: $v_{s_{m}}=\chi \omega_{s} \tau / m \pi$.

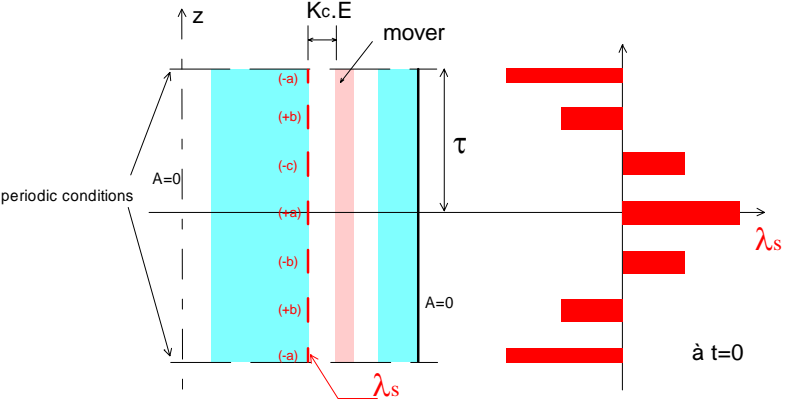

Figure 6: Slotted, infinite-length model

The expressions for the axial component of the magnetic field and the azimuthal component of the electric field, under these conditions, are, equations (14a) and (14b) :

$$
\begin{aligned}
& \mathrm{H}^{[\mathrm{n}]}{ }_{\mathrm{z}}(\mathrm{z}, \mathrm{r}, \mathrm{t})=\sum_{\mathrm{m}=1,5, \ldots}^{\infty} \frac{\gamma_{\mathrm{m}}{ }^{[n]}}{\mu^{[n]}}\left[\mathrm{X}_{\mathrm{m}}{ }^{[\mathrm{n}]} \cdot \mathrm{I}_{0}\left(\gamma_{\mathrm{m}}{ }^{[n]} \cdot \mathrm{r}\right)-\mathrm{Y}_{\mathrm{m}}{ }^{[\mathrm{n}]} \cdot \mathrm{K}_{0}\left(\gamma_{\mathrm{m}}{ }^{[\mathrm{n}]} \cdot \mathrm{r}\right)\right] \cdot \mathrm{e}^{\mathrm{j}\left(\mathrm{k}_{\mathrm{m}} \cdot \mathrm{z}+\chi \omega_{\mathrm{s}} \mathrm{t}\right)} \\
& \mathrm{E}^{[\mathrm{n}]}{ }_{\theta}(\mathrm{z}, \mathrm{r}, \mathrm{t})=\sum_{\mathrm{m}=1,5, \ldots}^{\infty} \mathrm{j} \chi \omega_{\mathrm{s}}\left[\mathrm{X}_{\mathrm{m}}{ }^{[\mathrm{n}]} \cdot \mathrm{I}_{1}\left(\gamma_{\mathrm{m}}{ }^{[\mathrm{n}]} \cdot \mathrm{r}\right)+\mathrm{Y}_{\mathrm{m}}{ }^{[\mathrm{n}]} \cdot \mathrm{K}_{1}\left(\gamma_{\mathrm{m}}{ }^{[\mathrm{n}]} \cdot \mathrm{r}\right)\right] \cdot \mathrm{e}^{\mathrm{j}\left(\mathrm{k}_{\mathrm{m}} \cdot \mathrm{z} \cdot \chi \omega_{\mathrm{s}} \mathrm{t}\right)}
\end{aligned}
$$

A comparison between the analytical model and finite element model is displayed in Figure 7 below, with results obtained for the same geometry as before. Here once again, a very strong level of correlation between the two models has been demonstrated.
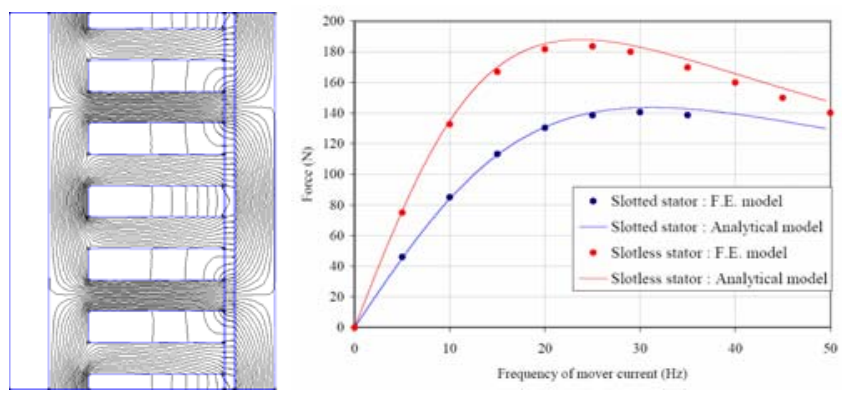

Figure 7: Flux density calculated using finite elements for $v=v_{s}(a)$, and the force developed for a slotted,

infinite-length machine vs. the rotor current frequency (b)

\section{B. Finite length analytical model}

In order to take account of the edge effects produced by the finite length of the stator, we have undertaken the same approach as before, yet this time in introducing a new amplitude $( \pm 1)$ modulation function $\Delta_{\mathrm{s}}$ of the current density $\lambda_{s}$ (see Fig. 8). The modulation length $L$, chosen arbitrarily, is such that: $L>>$.

$$
\Delta_{s}(z)=\sum_{\varsigma=1,3, . .}^{\infty} \frac{4}{\varsigma \cdot \pi} \sin \left(\frac{\varsigma \pi L}{L}\right) \cdot \cos \left(\frac{2 \varsigma \pi}{L} z\right)
$$

The expression of the modulated primary current then becomes (equation (16) :

$\lambda_{s}(z, t)=\hat{\lambda}_{s} \frac{12}{\pi^{2}} \sum_{\zeta=1,3, \ldots}^{\infty} \sum_{m=1,5, . .}^{\infty} \frac{1}{m \varsigma} \sin \left(\frac{m \pi}{12}\right) \sin \left(\frac{\varsigma \pi L}{L}\right)\left[e^{j\left(k_{m_{\zeta}}^{I} z+\chi \omega_{s} t\right)}+e^{j\left(k_{m s}^{I I} z+\chi \omega_{s} t\right)}\right]$ with: $k_{m \varsigma}{ }^{I}=\pi / \tau\left(m+\varsigma^{2 \tau} / L\right), k_{m \varsigma}{ }^{I I}=\pi / \tau\left(m-\varsigma^{2 \tau} / L\right)$ 


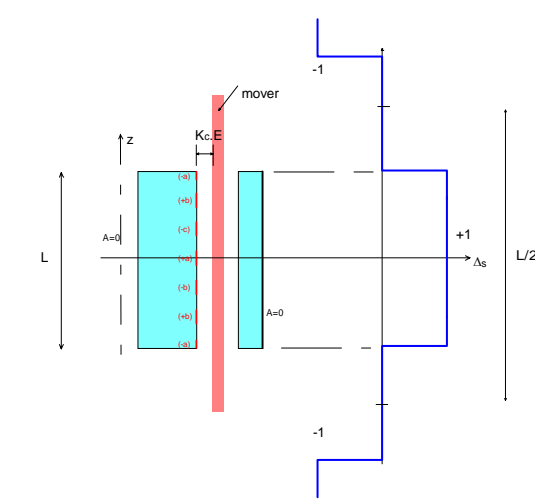

Figure 8: Slotted, finite-length model

Using the same equations as above, the expression derived for vector potential is, equation (17) :

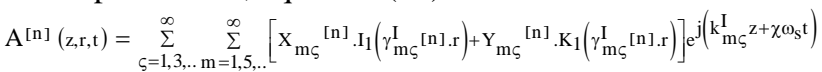

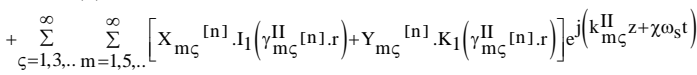

where: $\quad \gamma_{\mathrm{m} \varsigma}^{\mathrm{I}[\mathrm{n}]}=\sqrt{\mathrm{k}_{\mathrm{m} \varsigma}^{\mathrm{I}}{ }^{2}+\mathrm{j} \chi \omega_{\mathrm{s}} \mathrm{g}_{\mathrm{m} \varsigma}^{\left[{ }^{[\mathrm{n}]}\right.} \mu^{[\mathrm{n}]} \sigma^{[\mathrm{n}]}}$,

$$
\gamma_{\mathrm{m} \varsigma}^{\mathrm{II}}=\sqrt{\mathrm{k}_{\mathrm{m} \varsigma}^{\mathrm{II}} 2+\mathrm{j} \chi \omega_{\mathrm{s}} \mathrm{g}_{\mathrm{m} \varsigma}^{[I[\mathrm{n}]} \mu^{[\mathrm{n}]} \sigma^{[\mathrm{n}]}}
$$

$g_{m \varsigma}^{I}=1+\chi(g-1)\left(m+\varsigma \frac{2 \tau}{L}\right), g_{m \varsigma}^{I I}=1+\chi(g-1)\left(m-\varsigma \frac{2 \tau}{L}\right)$

Remark:

For $\tau / L \square 1$, we return to the case of an infinite-length machine.

The finite stator length also necessitates modulating the airgap permeance, which translates the increase in airgap at the generator edges. By applying the same modulation function as the one described above over the magnetic field, we obtain:

$\mathrm{H}_{\mathrm{z}}^{[\mathrm{n}]}(\mathrm{z}, \mathrm{r}, \mathrm{t})=\frac{\Delta_{\mathrm{s}}(\mathrm{z})}{\mu^{[\mathrm{n}]}}\left(\frac{\mathrm{A}^{[\mathrm{n}]}}{\mathrm{r}}+\frac{\partial \mathrm{A}^{[\mathrm{n}]}}{\partial \mathrm{r}}\right)$

where $A^{[n]}$ is given in equation (17).

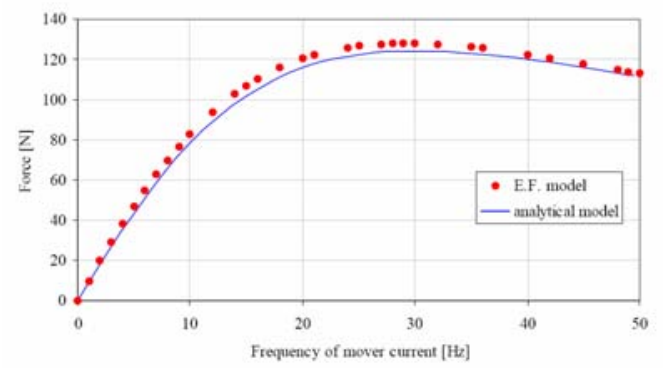

Figure 9: Force developed for a slotted,finite-length machine

Furthermore, the expression for electric field $E_{\theta}$ may be deduced directly from the vector potential expression: $E_{\theta}=j \omega_{\mathrm{s}} A$.

As shown in Figure 9 results, obtained using the same generator dimensions as before, the discrepancy between the two models remains relatively small.

An exhaustive study on the validity of the analytical model for various geometric parameters (with special emphasis on the ratio $\tau / L$ ) has been done. We show figure 10 the end effect reduction factor.

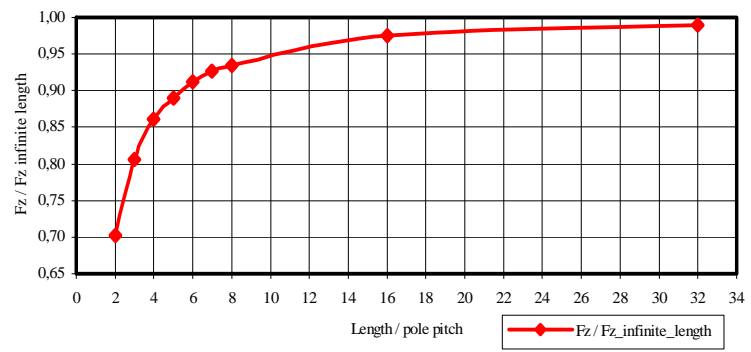

Figure 10 : Strength réduction versus (generator length / pole pitche)

\section{Schéma électrique equivalent}

Afin d'établir les équations électriques représentatives du générateur asynchrone et son schéma équivalent, il est nécessaire de calculer les différentes inductances et resistances primaire et secondaire.

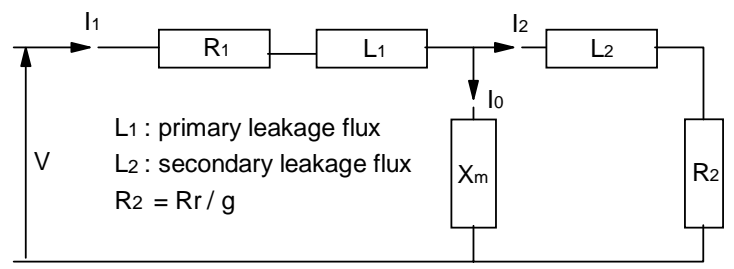

L’inductance et la résistance primaires d'une phase sont aisément définies :

$$
\begin{aligned}
& \mathrm{R}_{1}=p \cdot n^{2} \cdot \frac{\sigma \cdot \pi \cdot D}{S_{s}} \\
& \mathrm{~L}_{1}=\mu_{0} \cdot \pi \cdot \frac{n^{2}}{b}\left(\frac{D}{3} \cdot h+D_{m} \cdot e\right)
\end{aligned}
$$

With, p, length pitche pole number,
n, spire number,
b, coil thickness,
$\mathrm{D}$, mean coil diameter,
$\mathrm{D}_{\mathrm{m}}$, mean airgap diameter,
h, coil height,
e, airgap thickness
$\mathrm{S}_{\mathrm{e}}$, airgap surface, (airgap cut by $(\theta, \mathrm{r})$ plane)

Au secondaire, nous utilisons le modèle analytique. Il nous permet de connaître les puissances actives et réactives aux frontieres des regions, fer, entrefer, mover en integrant sur ces frontieres le vecteur de Poynting (voir paragraphe III.A.1). De ces puissances, nous pouvons déduire les impédances du secondaire, ainsi que l'impedance magnétisante en fonction de la fréquence des courants induits.

$\overline{P_{(s n)}}=\frac{1}{2} E_{\theta}\left(\gamma_{n} \cdot r_{n}\right) \cdot H_{z}^{*}\left(\gamma_{n} \cdot r_{n}\right) \cdot \mathrm{S}_{\mathrm{n}}$, 
et, $\overline{Q_{(s n)}}=\frac{1}{2} E_{\theta}\left(\gamma_{n} \cdot r_{n}\right) \cdot H_{z}\left(\gamma_{n} \cdot r_{n}\right) \cdot S_{n}$,

La Figure 11 présente les puissances active et réactive qui sont à l'origine des valeurs de R2 et L2.

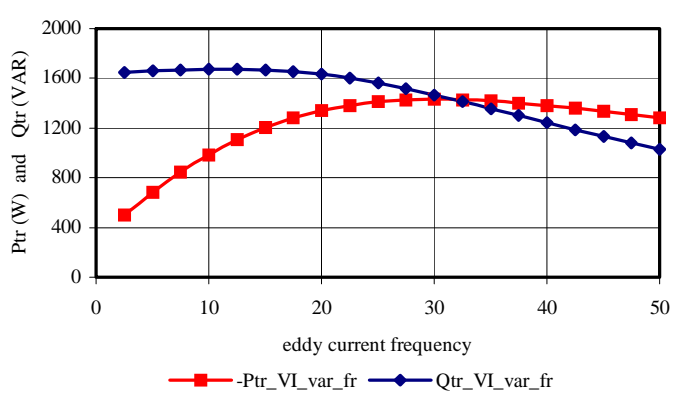

Figure 11:Ptr Qtr versus mover versus eddy currents frequency

\section{ETUDE GLOBALE DU COGÉNÉRATEUR}

Comme il est rappelé dans l'introduction, l'étude du cogénérateur a été partagé selon trois disciplines, électrotechnique, thermique et automatique. La modélisation du générateur présentée dans cette article est complétée d'une modélisation du moteur Stirling en vue d'optimiser globalement le cogénérateur et de le controller.

\section{A. Modélisation du moteur stirling}

La modélisation du moteur thermique est reprise des travaux de Julien Boucher [12].

Le cycle thermodynamique du moteur Stirling comporte deux isochores et deux isothermes. Par hypothèse, les échanges se réalisent en mode adiabatique. ( $\mathrm{P} . \mathrm{V}^{\gamma}=$ constante). Le moteur est à double effet, à piston libre.

Le point de fonctionnement du moteur se caractérise par la couple de valeurs $\left(y_{p \max }, f_{o s c}\right)$, où $y_{p \max }$ est l'amplitude de la course du piston et $f_{\text {osc }}$ sa fréquence d'oscillation. Ce point de fonctionnement $\left(y_{p \max }, f_{\text {osc }}\right)$ dépend de la différence de température entre les chambres chaude et froide. Ce point de fonctionnement impose, de facto, la puissance thermique fournie et la puissance électrique générée.

Par hypothèse, la variation de vitesse du piston et des déplaceurs est supposée sinusoïdale.

$$
\begin{aligned}
& y_{\text {pref }}=y_{p \max } \sin \left(2 \pi f_{\text {osc. }} t\right) \\
& y_{d 1}=y_{d \max } \cdot \sin \left(2 \pi f_{\text {osc }} \cdot t+\Phi\right) \\
& y_{d 1}=y_{d \max } \cdot \sin \left(2 \pi f_{\text {osc }} t+\Phi\right)
\end{aligned}
$$

Il s’en suit que, pour un point de fonctionnement donné, la position du piston est connue à chaque instant. Cette position sera la position-reference prise comme consigne de la commande du générateur $\left(y_{\text {ref }}\right)$.

L'équation d'état du moteur est de la forme (equation (21): $\left(\begin{array}{c}\ddot{y}_{p} \\ \ddot{y}_{d 1} \\ \ddot{y}_{d 2}\end{array}\right)=\left(\begin{array}{ccc}K_{p p} & K_{p d} & K_{p d} \\ K_{d p} & K_{d d} & 0 \\ K_{d p} & 0 & K_{d d}\end{array}\right)\left(\begin{array}{c}y_{p} \\ y_{d 1} \\ y_{d 2}\end{array}\right)+\left(\begin{array}{ccc}D_{p p} & 0 & 0 \\ D_{d p} & D_{d d} & 0 \\ D_{d p} & 0 & D_{d d}\end{array}\right)\left(\begin{array}{c}\dot{y}_{p} \\ \dot{y}_{d 1} \\ \dot{y}_{d 2}\end{array}\right)+\left(\begin{array}{c}1 / m_{p} \\ 0 \\ 0\end{array}\right) F_{g e ́ n e ́}$

Ce modèle d'état s'apparente à un ressort couplé à un élément visqueux. Nous pouvons en déduire que le moteur Stirling se comportera comme un oscillateur si la force appliquée par le générateur, compense le terme visqueux. Nous obtenons alors l'équation de base d'un oscillateur: $\ddot{y}_{p}=k y_{p}$, équation représentative d'un fonctionnement 'à piston libre', en particulier.

\section{B. Contrôle-commande du cogénérateur}

Le contrôle-commande et l'optimisation globale du cogénérateur sont repris des travaux d'Isabel Garcia Burrel [11].

Le fort couplage entre le moteur Stirling et le générateur électrique nécessite de les 'contrôler' simultanément. La grandeur nous permettant d'assurer le pilotage de l'ensemble en boucle fermée est la position du piston, pièce commune au moteur et au générateur

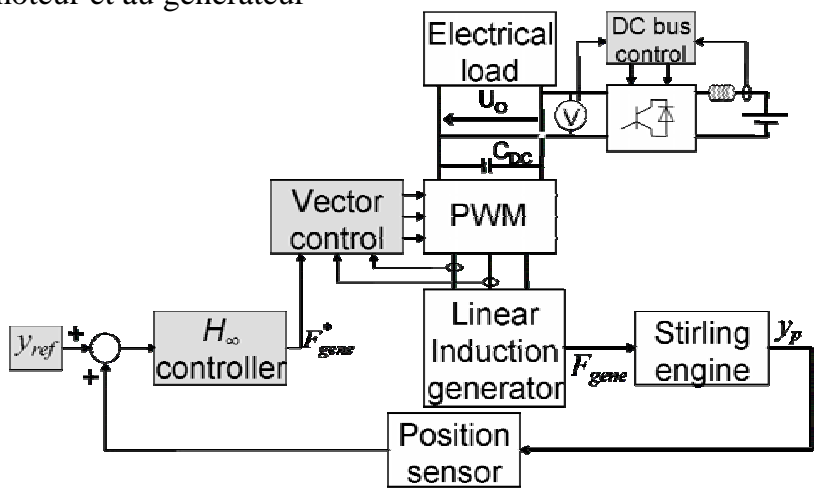

Figure 12: cogenerator control-command

Comme l'exprime l'équation d'état du modèle thermique, ce contrôle a pour but d'assurer, à tout instant, l'équilibre entre l'effort moteur (moteur Stirling) et l'effort résistant (générateur asynchrone), équilibre indispensable au fonctionnement 'à piston libre'.

Comme nous l'avons vu au paragraphe IV.A, c'est cette position du piston qui est la consigne entrée pour contrôler le générateur. Ce contrôle est de type vectoriel.

\section{Optimisation globale du cogénérateur}

Cette etude se devait d'être complétée par une demarche d'optimisation globale, il est evident que l'optimisation de chacun des composants pris isolément ne conduit pas nécessairement à l’optimisation du cogénérateur pris dans son ensemble.

Cette optimisation avait pour but de répondre à deux objectifs

- minimiser les pertes du générateur.

- minimiser la taille des composants de l’onduleur. 
L’optimisation a été réalisée avec un logiciel utilisant un algorithme génétique NSGA II. L'avantage d'un tel algorithme est la possibilité de prendre en compte un grand nombre de paramètres, associés à des objectifs et des contraintes multiples, ces objectifs et ces contraintes étant calculés avec les modèles électriques et thermiquesprécedemment décrits.

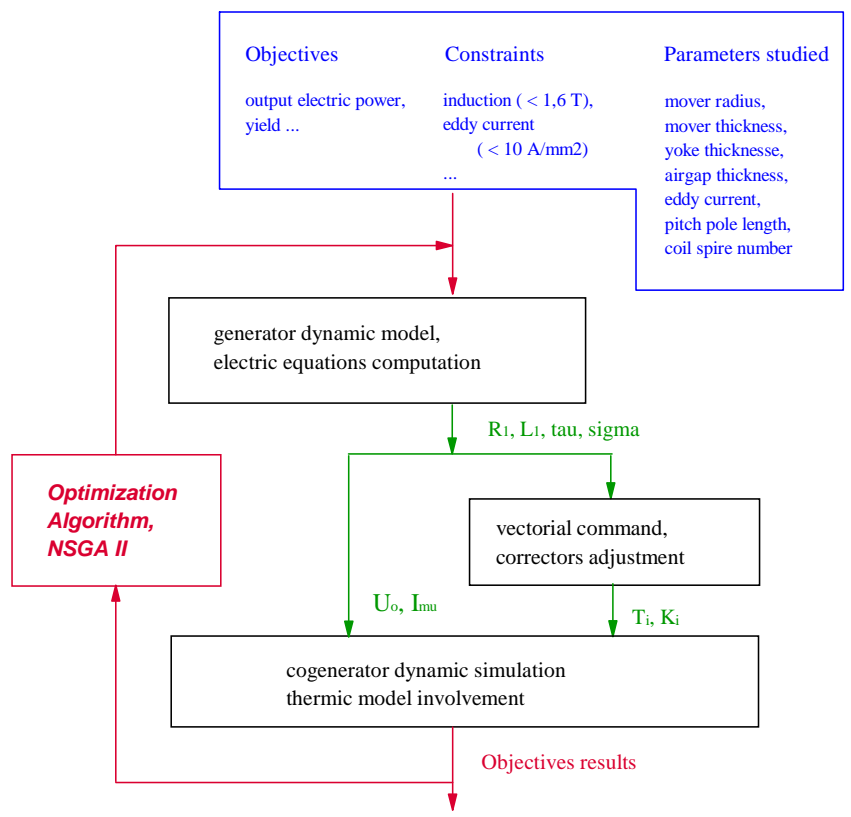

Figure 13: Optimisation process

La figure [14] donne un exemple de résultat d'optimisation. Il concerne la minimization des pertes dans le générateur et la minimization de la taille des semi-conducteurs de l'onduleur.

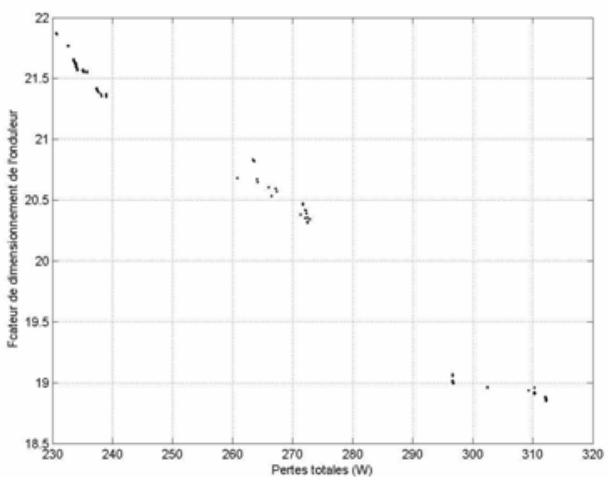

Figure 14 : looses:generator reduction and electronic components inverter optimization

\section{CONCLUSION}

This article has set forth the steps behind development of a 3D analytical model for the tubular linear induction generator with inclusion of both slot and edge effects. Utilizing a frequency-based approach, this model proves to be relatively accurate with respect to the finite element model. Besides calculating the electromagnetic performance of the generator, our new model makes it possible to identify elements of equivalent dynamic electrical diagram [9].

Also, this article highlights the multi-objective optimization studies conducted within the specific scope of co-generation operations [11] through applying a genetic algorithm [13].

\section{REFERENCES}

[1] H.I. Onovwiona, V.I. Ugursal, "Residential cogeneration systems: Review of current technology", Renewable and sustainable energy review, 2004 (Online) available: www.sciencedirect.com.

[2] P. François, L. Prévond, G. Descombes, International Patent N PCTFR02-00173, 2002.

[3] S. Backhaus, G.W. Swift, "A thermoacoustic Stirling heat engine, detailed study”, J. Acoust. Soc. Am. Proc. 107 (6), 2000, CD-ROM.

[4] B. Alvarenga, I. Chabu, J.R. Cardoso, "Modeling and testing of a Ring cage tubular linear induction motor for an oil pumping system”, 1993.

[5] A.L. Cullen, TH. Barton, "A simplified electromagnetic theory of the induction motor, using the concept of wave impedance", IEE Proceedings, Vol. 105, Part C, No. 8, pp. 331-336, September 1958.

[6] M.V. Zagirnyak, R.M. Pai, S.A. Nasar, "Analysis of tubular linear induction motors, using the concept of surface impedance”, IEEE Transactions on Magnetics, Vol. MAG-21, N04, pp.1310-1313, July 1985.

[7] F.W. Carter, "Note on air gap and interpolar induction”, J. IEE, 1929.

[8] A. Balakrishnan, W.T. Joines and T.G. Wilson, "Air gap reluctance and inductance calculation for magnetic circuits using a SchwarzChristopheffel transformation”, IEEE trans. On Power Electronics, Vol. 12, $\mathrm{N}^{\circ} 4,1997$.

[9] R.M. Pai, I. Boldea, S.A. Nasar, "A complete equivalent circuit of a linear induction motor with sheet secondary", IEEE Trans. On Magnetics, Vol. 24, N²1, January 1988, pp. 639-654.

[10] K. Deb, A. Pratap, S. Agarwal, T. Meyarivan, “A fast and elitist multiobjective genetic algorithm: NSGA-II, IEEE Trans. On Evolutionary Computation, vol. 6, Issue 2, April 2002, pp. 182-197.

[11] I. Garcia Burel, L. Prévond, S. Le Ballois, E. Monmasson, "A Stirling micro-cogenerator emulator”, IECON Proc., Paris, November 2006.

[12] P. Nika, J.Boucher, F. Lanzetta, P. François, L. Prévond, B. Multon, 30mai-2juin 2005. "La cogénération Stirling à pistons libres”, Congrés Français de Thermique, SFT 2005, Reims

[13] K. Deb, A. Pratap, S. Agarwal, T. Meyarivan, "A fast and elitist multiobjective genetic algorithm: NSGA-II, IEEE Trans. On Evolutionary Computation, Vol. 6, Issue 2, April 2002, pp. 182-197. 